\title{
Study of dose rate in the brain model based on the neutron beam of SUT-MNSR
}

\author{
K aijian Li, A yut Limphirat, Nuanwan Sanguansak \\ Suranaree U niversity of Technology,N akhon Ratchasima,30000, Thailand)
}

\begin{abstract}
Boron neutron capture therapy (BNCT) is tumor-cell targeted radiotherapy that has significant superiority over conventional radiotherapies. The most neutron beams used for BNCT are from the reactors with high power, new design M iniature Neutron Source Reactor(M NSR) with $45 \mathrm{~kW}$ with BNCT beam for Suranaree University of Technology(SUT) is being designed and built. According to SUT-M NSR physics design, SUT-M NSR will have the epithermal neutron beam for BNCT treatment. The dose rate distribution in the body should be estimated before SUT-M NSR is used for BNCT clinical trials (B rain tumor). This paper introduces the simulation for SUT-M NSR neutron beam by M onte Carlo N-Particle Transport C ode (M CNP), and the establishment of human brain model and physics dose rate distribution in brain tumor by MCNP program.The brain model is established according to the different element in the skin, skull and tissue, the distribution of neutron dose and Gamma dose in the brain model were calculated.
\end{abstract}

\section{Introduction}

Since Japanese scientists treated a patient with head and neck cancer with BNCT in1960s, so far, some clinical trials used by BNCT have been finished by reactor neutron source. SUT-MNSR is specially designed nuclear reactor and used for Boron Neutron Capture Therapy (BNCT), especially for malignancies and cancers treatment. Thermal power of the reactor is $45 \mathrm{~kW}, \mathrm{UO}_{2}$ as fuel, and with metallic beryllium as reflector. The fission heat produced by the reactor is removed by the natural circulation. On the bottom of the reactor core, there are two neutron beams which are used for BNCT, one is thermal neutron beam, other is epithermal neutron beam, the neutron spectrum can be shifted by changing the thickness of water. Therefore, one beam has two kinds of neutron flux (thermal neutron flux and epithermal neutron flux), two neutron beams are used for the treatment of different kinds of cancer by BNCT, the diameter of beam exit is $12 \mathrm{~cm}$. The simulation for SUT-MNSR neutron beams is done by Monte Carlo N-Particle Transport Code (MCNP), the parameters at beam exit meets IAEA ( Barth et al., 2005) standard of BNCT. See Fig 1. The epithermal neutron beam of SUT-MNSR will be used for brain glioma treatment. The purpose of this paper is to investigate the dose rate distribution in brain model based on the epithermal neutron beam of SUT-MNSR.

\section{Material and Methods}

The main calculating parameters at the epithermal neutron beam exit are that the epithermal neutron flux rate at the exit is $1.30 \times 10^{9} \mathrm{n}^{\bullet} \mathrm{cm}^{-2} \bullet \mathrm{n}^{-1}$, the ratio of Gamma dose

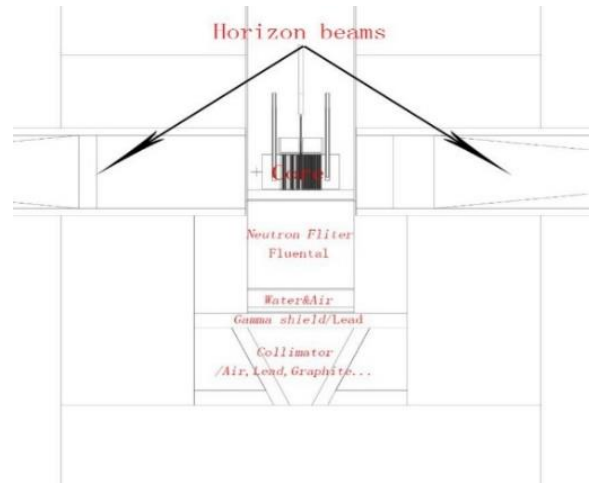

Fig. 1. SUT-M NSR vertical neutron beam for BNCT

rate to epithermal neutron flux rate is $3.70 \times 10^{-14}$ $\mathrm{Gy} \cdot \mathrm{cm}^{2} \bullet \mathrm{n}^{-1}$, the ratio of thermal neutron flux rate to epithermal neutron is 0.056. Compared with the IAEA recommended beam parameters for BNCT, the epithermal beam quality of SUT-MNSR with $45 \mathrm{~kW}$ is accredited.

Snyder Brain Model (Deng Li et al., 2011) was chosen for brain dose rate distribution Monte Carlo simulation. Snyder brain model with three regions has brain tissue, skull and skin from the inner to outer of model, their compositions of brain tissue, skull and skin are based on the ICRU 46.report (1992).

In order to compare the dose rate results in the brain model with Boron or without boron, one of Snyder Brain Model boron compound is presumed to be $10 \mathrm{ppm}$ in the normal tissue and $40 \mathrm{ppm}$ in tumor $(\mathrm{H}$, Kumada et al., 2007). It is assumed that the boron compound will not be uptaken by the bone and skin.

\footnotetext{
Corresponding author: 18500184136@163.com
} 
The other one of Snyder Brain Model only carries normal tissue, worked for control group. See Figure 2(Left with Boron, Right without boron), the epithermal neutron comes from the top direction. For the left brain model, the tumour volume is $\approx 3.1 \mathrm{~cm}^{3}$, the depth id $1.75 \mathrm{~cm}$ from the surface of skin and the maximum width is $1 \mathrm{~cm}$.

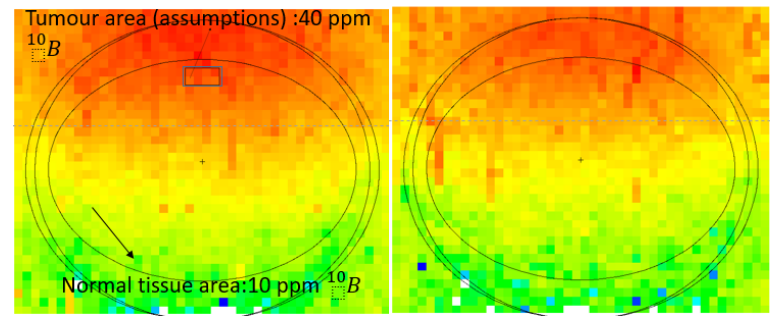

Fig. 2. Snyder Brain Model with Boron(left ) and without Boron (right)

The radiobiological characteristics of different dose components (low-LET $\gamma$-rays dose rate, neutron dose rate and boron dose rate) of BNCT exposure are examined in the model. Biological factors are widely used in the calculation of dose rate (Gy-Eq) of Snyder brain model. During the treatment, the dose limits of eye, spinal cord and skin are 5 Gy-Eq, $10 \mathrm{~Gy}$-Eq and 11 Gy-Eq respectively(Yu H T et al., 2011). The absorb equivalent dose rate is determinted by the compound biological effectiveness (CBE) factors and relative biological effectiveness (RBE), the RBEN and RBEY are set to 3.2 for neutron and 1 for gamma ray respectively(Yu H T et al., 2011), the CBE are set 1.3 for normal tissue and 3.8 for tumor (Coderre et al., 1999). Total dose Dtotal in Gy-Eq unit can be determined according to equation:

$$
\mathrm{D}_{\text {total }}=\mathrm{CBE}_{\mathrm{B}} \cdot \mathrm{D}_{\mathrm{B}}+\mathrm{RBE}_{\mathrm{N}} \cdot \mathrm{D}_{\mathrm{N}}+\mathrm{RBE}_{\gamma} \cdot \mathrm{D}_{\gamma}
$$

Where, $D_{B}, D_{N}$ and $D_{\gamma}$ are physical dose results.

\section{Material and Methods}

MCNP code has been performed to investigate the beam quality at SUT-MNSR epithermal neutron beam exit. As mentioned earlier, SUT-MNSR neutron spectrum can be changed from thermal neutron beam to epithermal neutron (with the fast neutron contamination) by changing thickness of water. See Figure 3.

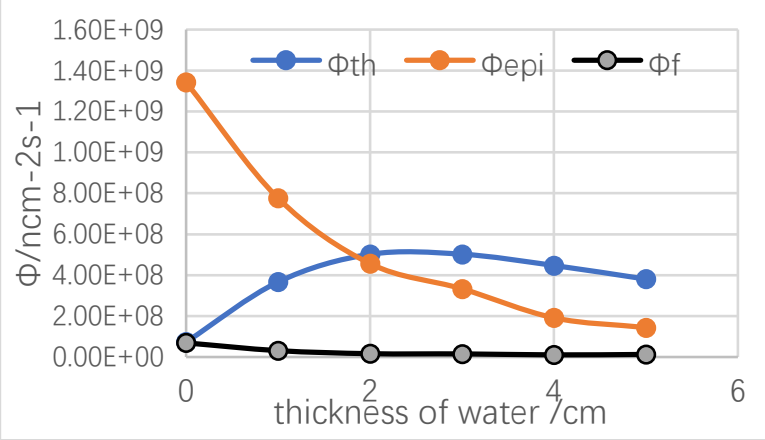

Fig.3. Neutron spectrum change with the thickness of water
The same irradiation condition simulations are analyzed for two Snyder brain models. The maximal value of neutron dose rate at skin is $0.248 \mathrm{~Gy} / \mathrm{min}-\mathrm{Eq}$ ( Fig. 4 and Fig.5).

The gamma dose is mainly from reactor core contamination and hydrogen and nitrogen neutron capture gamma reaction and boron neutron capture reaction $(0.477 \mathrm{MeV})$ or other reaction but can be ignored. The gamma contamination result by F5: $\mathrm{p}$ tally card at BNCT neutron beam exit is estimated, it is 7.3 $\times 10^{-14} \mathrm{~Gy} \mathrm{~cm}^{2} \mathrm{n}^{-1}$ (Statistical errors $\leq 6 \%$ ). IAEA suggests value at exit of facility is $\leq 13 \times$ $10^{-13} \mathrm{~Gy} \mathrm{~cm}^{2} \mathrm{n}^{-1}$.

In the absorbed dose result, it is obvious that the energy deposition value of brain model with boron in tumour area is much higher than brain model without boron at the same area. And the value of brain model with boron at normal area is higher than brain model without boron at the same area, the reason is that thermal neutron boron neutron capture reactions occured in tumour area. This result shows that SUTMNSR will be successful for BNCT.

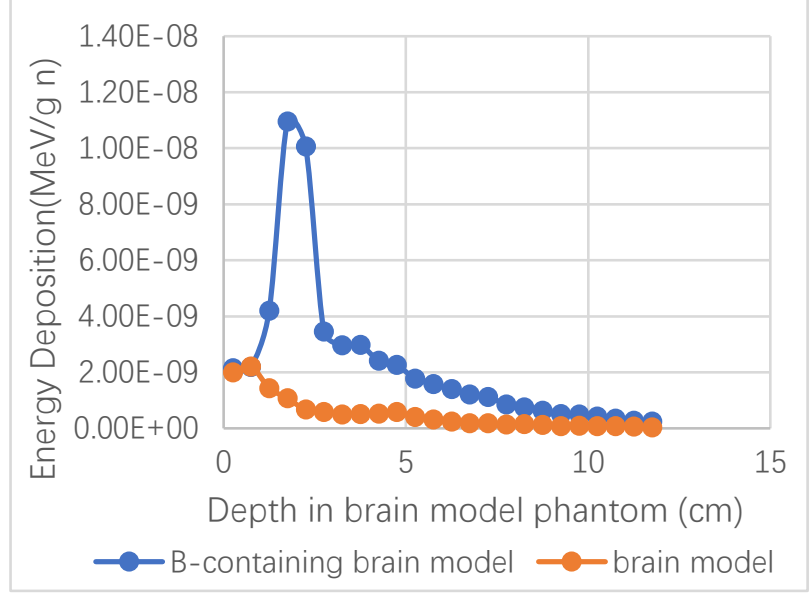

Fig. 4. Energy deposition change by depth

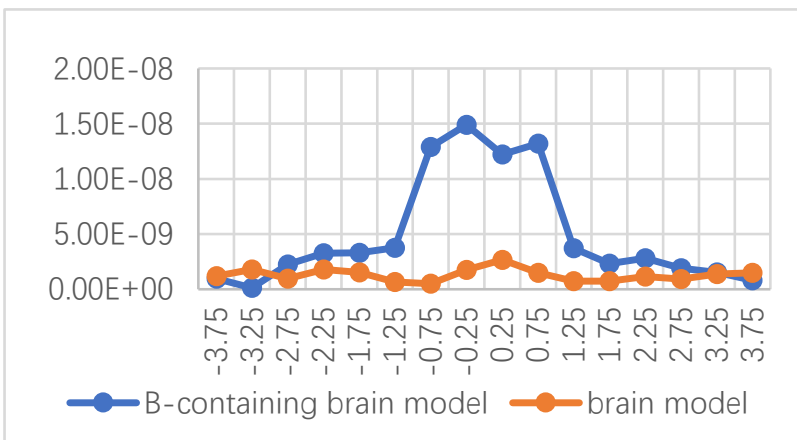

Fig.5. Energy deposition horizontal cross-section (depth in brain model $2 \mathrm{~cm}$-tumour area).

\section{Conclusions and futures work}

The Snyder brain model has been established according to the different composition of brain tissue, skull and skin, and the dose rate distribution in the two brain models is researched. The irradiation time at SUTM NSR neutron beam exit can be kept less then $1 \mathrm{hr}$ 
according to the absorb dose results. The CPU time will be around 24hrs for each brain model, the Monte Carlo statistical errors at important part is $\leq 6 \%$ after enhanced the "cps". The dose simulation study in this paper is based on assumption that the boron concentration is 40ppm in the tumour area and 10ppm in the normal tissue. But according to Finland's clinical trails (Ryynanen, et al., 2000), the patient injection with boron drug (BPA) is done two hours before irradiation, the boron concentrate rate will drop quickly during the irradiation, which makes the dose prediction quite difficulty. On the other hand, J apan indicated that the continuous injection of boron drug (BPA) (Ono et al., 2006) during irradiation will be able to keep the boron concentrate rate in the blood and make the dose prediction more accurate. The feasibility study is currently being investigated for SUT-MNSR BNCT facility.

\section{References}

1. B arth, R. F., Coderre, J. A ., Vicente, M. G. H. and Blue, $T$.

E. (2005). Boron neutron capture therapy of cancer current status and future prospects. Clinical Cancer Research. 11(11): 3987-4002

2. Deng, Li, et al. Diagnostic Techniques and Surgical $M$ anagement of B rain Tumors. In Tech, 2011

3. H, K umada., and K, Y amamoto., et al., D evelopment of JCDS, a computational dosimetry system at JAEA for boron neutron capture therapy. Journal of Physics: Conference Series.74(2007)

4. Report I. Photon, Electron, Proton and Neutron Interaction Data for B ody Tissues[M ],Photon, el ectron, proton, and neutron interaction data for body tissues. International Commission on Radiation Units and M easurements, 1992

5. $Y u H T$, Liu $Y W H$, Lin $T Y$, et al. BNCT treatment planning of recurrent head-and-neck cancer using THOR plan[J]. A pplied Radiation \& Isotopes Including $D$ ata Instrumentation $\&$ M ethods for $U$ se in A griculture Industry \& Medicine, 69(12):1907-1910 (2011)

6. Ryynanen M .P., K ortesniemi M ., et al ., 2000. M odel for estimation of the 10B concentration after BPA -fructose complex infusion in patients during epithermal neutron irradiation in BNCT., A ppl.Radiat. Oncol. Biol. Phys., 48, S1145-S1154.

7. O no K., M asunaga S.I ., et al., N eutron irradiation under continuous BPA injection for solving the problem of heterogeneous distribution of BPA ., ICN CT-12, Japan. (2006) 